\title{
Kinematic study and implementation of a bio-inspired robotic fish underwater vehicle in a Lighthill mathematical framework
}

\author{
Abhra Roy Chowdhury ${ }^{1 *}$, Vinoth Kumar ${ }^{1}$, Bhuneshwar Prasad ${ }^{1}$, Rajesh Kumar ${ }^{2}$ and Sanjib Kumar Panda ${ }^{1}$
}

\begin{abstract}
This paper has focused on the formulation of the biological fish propulsion mechanism given by Sir J. Lighthill mathematical slender body theory for a bio-inspired robotic fish. A 2-joint, 3-link multibody vehicle model biologically inspired by a body caudal fin (BCF) carangiform fish propulsion is designed. The objective is to investigate and show that a machine mimicking real fish behavior can navigate efficiently over a given distance with a good balance of speed and maneuverability. The robotic fish model (kinematics and dynamics) is integrated with the Lighthill (LH) mathematical model framework. Different mathematical propulsive waveforms are combined with an inverse kinematics-based approach for generating fish body motion. Comparative studies are undertaken among a non-LH model, a LH model, and the proposed propulsive wave models based on a distance-based performance index. Proposed LH cubic and NURB quadratic functions are found to be $16.32 \%$ and $17.94 \%$ efficient than a non-LH function, respectively. With the help of the simulation results, closed-loop experiments are done and an operating region is established for critical kinematic parameters tail-beat frequency and propulsive wavelength. The simulation and experimental plots are compared and found to be similar to the kinematic behavior study of the biological yellowfin tuna.
\end{abstract}

Keywords: Biology inspired robotics; Body caudal fin; Lagrange-Euler equations; Lighthill equation; Operating region

\section{Background}

Biomimetics [1] reflects the features and capabilities of the biological evolution [2] of a system that could be efficiently replicated or mimicked in a human engineered system to the design of new technologies and the improvement of conventional ones. This approach has been proposed to be the answer to the improved performance and reliability for large scale complex systems by faster adaptation with dynamic environment. One of the focused technologies has been the development of autonomous underwater vehicles [3] as a greater part to the increasing interest in unmanned underwater surveillance and monitoring. Of particular interests are regions of the underwater environment which are unexplored and dynamic as well as underwater detection, pollution source tracking, underwater archeology, search and

\footnotetext{
* Correspondence: abhra.roychowdhury@nus.edu.sg

'Department of Electrical and Computer Engineering, National University of Singapore, Singapore 117576, Singapore

Full list of author information is available at the end of the article
}

rescue, and so forth. The study of underwater evolution of life and its plethora of locomotion modes has long been a subject of interest to the biological community. Majority of conventional design of autonomous underwater vehicles used propellers as their principal mode of propulsion. The propeller-based locomotion [3], although rendered the initial answers to underwater locomotion, set issues on high-maneuverability, efficiency, and low power consumption. The scientific community and researchers also found that propeller-strikes produce greater amount of marine debris, marine creature's mortality and shallow water ecosystem disturbances. Biomimicked or fish-like robots are expected to be quieter, more maneuverable (lesser accidents), and possibly, more energy efficient (longer missions). Undulating-finned robot can preserve undisturbed condition of its surroundings for data acquisition and exploration (stealth). The movement of fish through water without creating ripples and eddies were more reasons to choose a bio-inspired design for underwater locomotion. 
Considering the propulsive features [4] of existing fish modes, a novel propulsive mechanism that integrates fishlike swimming with modular links and fin movements has been proposed [3] where the modeling, simulation, and development studies of a body caudal fin (BCF) [5] carangiform-based prototype is built and tested. The robot will be able to implement speedy and efficient fish-like swimming. The present research work specifically identifies the usefulness of present model for purposes of both speed and maneuverability. The kinematics-based approach allows producing a dynamic body motion that can reproduce the fluid flow pressure field generating the undulatory motion of the fish. Further, the kinematics and dynamics study helped to frame the mathematical formulation of the fish body motion describing its dynamic behavior. From a robotics perspective, defining and enhancing the swimming efficiency is still a kernel issue in the study of robotic fishes. However, the fish robot swimming like a real fish does not guarantee that it would achieve the same high efficiency [3]. The undulatory (oscillatory) nature of the fish motion has been prominently mentioned in several works [4]. Another solution adopted by researchers [5-8] is to conduct large number of experiments and find empirical expressions to refine the body's motion functions and fin propulsion. Due to the complex nature of the mechanical system, the paper focuses on developing a linear system model using robot dynamics derivation. The simulation environment is in MATLAB๑, Simulink $\odot$, and SolidWorks $\odot$. The contributions in the present work are enumerated as following:

- Mathematical input waveforms are proposed in LH framework to generate different types of (undulatory/ oscillatory) body propulsive waves. Comparative study of each model with the fundamental LH quadratic wave model is done for a performance parameter based on the total trajectory length.

- Each of the bio-inspired wave function combines with an inverse kinematics-led trajectory planning resulting in a bio-inspired algorithm to produce laterally compressed waveforms of the caudal region.

- Integrate the present robotic fish mathematical model (kinematics and dynamics) with the proposed bio-inspired algorithms resulting from different mathematical inputs.

- Finding the operating region (ORE) for the identified kinematic parameters to facilitate closed-loop control based on the characterization of the biological fish swimming model.

The underlying subject of this research is to explore the remarkable ability of mathematical (applied mechanics) and physical concepts leading to fundamental insights behind fish biology. It has been found that sophisticated arithmetic calculations can bring practical benefits in revealing the fundamental biological systems. Darwinianinspired evolution in modeling of biological systems is a strong partner of experimental work in physical sciences based on the principles in Newtonian and quantum frames. Present research investigates into this idea. The limitation of the paper is the linearized model of the robotic fish to undertake the study. Another assumption being made is stationary fluid, and therefore, the forces acting on a single link are due to the motion of that link. This may introduce inaccuracies in the manipulator model, while the discretized algorithm is implemented in a digital computer. The paper is organized as follows. In the 'System model' section, the design characteristics and features of the prototype are explained with the kinematics and dynamics modeling studies of a three link robotic fish. The 'Lighthill mathematical framework design' section discusses the Lighthill's existing mathematical framework and its integration with the present prototype. This section also investigates the proposal and study of the different mathematical input waveforms generating the fish undulatory and oscillatory motion. The 'Results and discussion' section presents the robotic fish body undulatory propulsion mechanism for a given trajectory, simulation, and experimental results with/without the Lighthill wave function as well as with different mathematical input in LH framework. Comparisons are based on a distancebased performance index. Closed-loop experiments [9] were made on the basis of comprehensive kinematic analysis governing the fish undulatory motion. An operating region is found for the dominant kinematic parameter TBF and propulsive wavelength each to facilitate future experiments for such bio-inspired systems. It is also verified with the reported literature for the real fish motion. In the 'Conclusions' the conclusions and directions for future work are discussed.

\section{Methods}

\section{System model}

The BCF mode carangiform style [5] of locomotion shown in Figure 1 is approximated using a 3-link (including the pectorals attached to the head) mechanism with two actuated joints as illustrated in Figure 2. The first link as the 'head' and second link as the body are roughly two-thirds of the length of the entire robot. The tail of the robot is formed by the third link connected to the caudal fin. The configuration of the robotic fish and non-fixed head is illustrated. Our 3-link mechanism $(n=2)$ is a reasonable approximation to the BCF carangiform locomotion, and therefore, small modifications of this model should have been used in the analysis of carangiform swimming. Moreover, the caudal peduncle and fin have been rendered in to a thunniform (shark) semi-crescent structure for an 


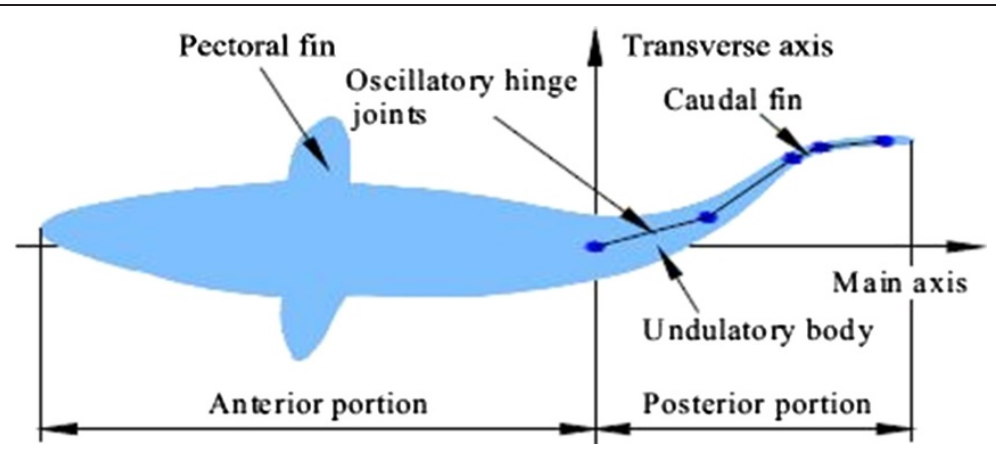

Figure 1 BCF mode of carangiform swimming showing undulatory motion in one-third part of the posterior body.

efficient thrust and therefore allowing high cruising speed for longer period of time [5]. While the kinematics study explains the geometry of the motion of robotic fish w.r.t, a fixed reference coordinate frame as a function of time, the dynamics of any rigid body [10-12] can be completely described by the translation of the centroid and the rotation of the body about its centroid. This leads to the ability to derive the actuator torques necessary to produce the tail motion that is desired. A linearized kinematic and dynamic model of the robotic fish system is developed. The present research work investigates into the system modeling as an $n$ joint manipulator-based mobile vehicle; the earth-fixed frame has been defined w.r.t. the fixed body reference

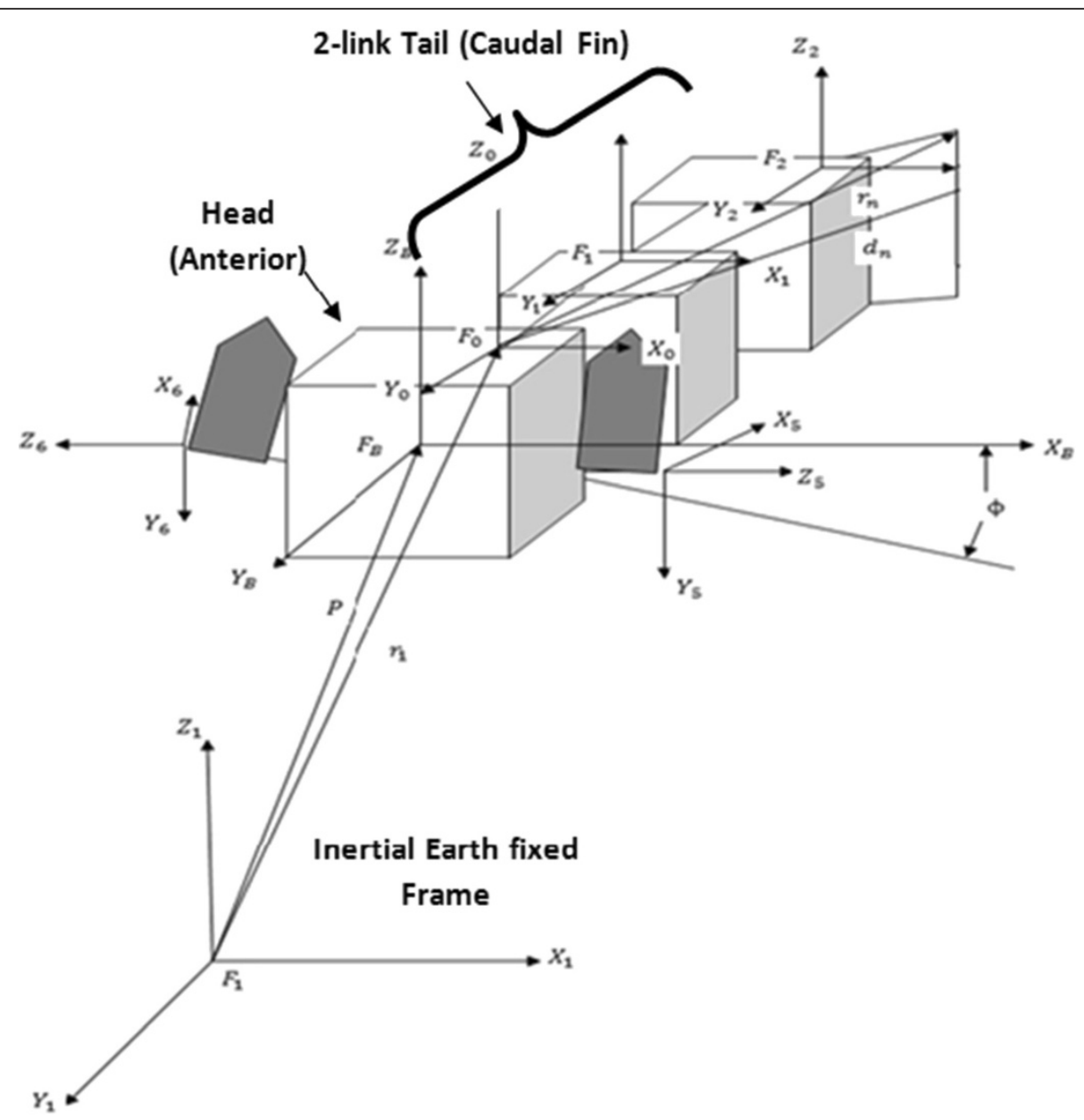

Figure 2 Relative orientations and locations of local coordinate frames at CM of the head and inertial earth-fixed reference frame. 
frame. Relevantly, the two major sections of the present paper robotic fish system model are the following:

- Denavit-Hartenberg (DH) kinematics model [11]: The kinematics due to translation and rotation along the joints on the robotic fish system in fluid (water) environment.

- Lagrangian dynamics model $[10,11]$ : The dynamics due to kinetic and potential energies generate along each link and inertia of the free-flow water entrained in the robotic fish system.

When describing the kinematics and dynamics of the model shown in Figure 2, the interlink actuator shaft constitutes the inertial frame of reference. A local coordinate frame is assigned to each degree of freedom (DOF). The coordinate frames are assigned according to the standard DenavitHartenberg notation [11] mentioned in Appendix A.

\section{Generalized equations of motion}

The dynamic equations of the robotic fish are obtained using the Lagrange-Euler formulation $[10,11]$ given as:

$$
\frac{d}{d t}\left(\frac{\partial L}{\partial \dot{q}_{i}}\right)-\frac{\partial L}{\partial q_{i}}=\tau_{i} \quad i=1,2, \ldots, n
$$

The Lagrange (L) function is defined as the difference between the kinematic and potential energies expressed as:

$$
L=K-P
$$

where $K$ is the total kinetic energy of the robot; $P$ is the total potential energy of the robot; $q_{i}$ is the joint variable of $i_{t h}$ coordinates; $\dot{q}_{i}$ is the first time derivative of the $i_{t h}$ joint variable, and $\tau_{i}$ is the corresponding generalized force (external torque) at $i_{t h}$ joint acting on the head. The manipulator dynamic equations have been developed in three dimensions for an $n$-link manipulator on a 6-DOF base, assuming that there is gravity acting on the system. The equations of motion for the present 2-link robotic fish based on the $n$-link serial manipulator can be set as:

$$
\sum_{\substack{j=1 \\ i=1,2 \ldots, n}}^{n} D_{i j}(q) \ddot{q}_{j}+\sum_{k=1}^{n} \sum_{m=1}^{n} h_{i k m} \dot{q}_{k} \dot{q}_{m}+c_{i}=\tau_{i}
$$

where $D(q)$ is the symmetric inertia matrix; $h(q, \dot{q})$ is the velocity coupling vector or Coriolis and centrifugal force vector; $c(q)$ is the gravitational vector; and $\tau$ is the generalized force of the Lagrange equations. As the above equation has been successfully used for investigating the dynamics of underwater vehicles $[10,12]$ as well as robotic manipulators [11], this research therefore aims to develop an $n$-joint manipulator-based mobile vehicle. Following the analysis, the mechanical design in SolidWorks is implemented. SolidWorks as the mainstream software in virtual prototype field combines multibody dynamics modeling with large displacements as well as multifunctioning tools. It has a more powerful geometric modeling function. By utilizing SolidWorks, a kinematics model of a robotic fish, with coordinated motion of multiple propulsive mechanisms, is established as shown in Figure 3a,b,c and the real hardware prototype used for closed-loop experiments [9] is shown in Figure 3d. Details of the dynamic modeling of present bio-inspired robot can be found in [12].

\section{Lighthill mathematical framework design}

The initial work to understand the fish kinematics appeared prominently in the article by James Gray [13]. It mentions about the fish swimming by generating a traveling wave down their bodies from the anterior (leading surface) toward the posterior caudal tail (trailing surface). This propulsive wave travels faster than the fish body (center of mass) velocity. The wave amplitude also increases from the head to the tail, maximum at the narrow peduncle region. Lighthill in his analysis of Gray's work [13] extended and formulated the slender body theory, which proposed the fact that the overall fluid flow around the body is a compound of the steady flow around the straight body and the flow due to the lateral displacement $h(x, t)$. The second component (flow) $V(x, t)$ for a given cross sectional area $S_{x}$ with fluid velocity $U$ given by:

$$
V(x, t)=\left(\frac{\partial h}{\partial t}\right)+U\left(\frac{\partial h}{\partial x}\right)
$$

Lighthill's theory discusses the swimming efficiency in detail and discusses on the role of flow produced by the lateral displacement on the efficiency improvement during the undulatory propulsion. The overall swimming efficiency as a function of overall fluid flow $V(x, t)$ is termed as Froude's efficiency [13] $\eta_{f}$ given by:

$$
\eta_{f}=1-\frac{1}{2}\left[\frac{V(x, \bar{t})_{x=l}^{2}}{\left(\frac{\partial h}{\partial t}\right) V(x, t)_{x=l}}\right]
$$

The theory uses a set of partial differential equations to calculate the thrust and efficiency of swimming with definite rhythmic and symmetric body motions. Supporting a laterally compressed wave and maintaining a high Froude number efficiency (as might be done by the biological fishes in real), the motion of a traveling wave displacement vector $h(x, t)$ was introduced by Lighthill as an empirical expression given by:

$$
h(x, t)=f(x) \times g(t-x / c)
$$

where the $f(x)$ term that indicates the amplitude, $g(x, t)$ is an oscillatory frequency dependent wave function, and $c$ is the fish body velocity. Out of the many alternative 

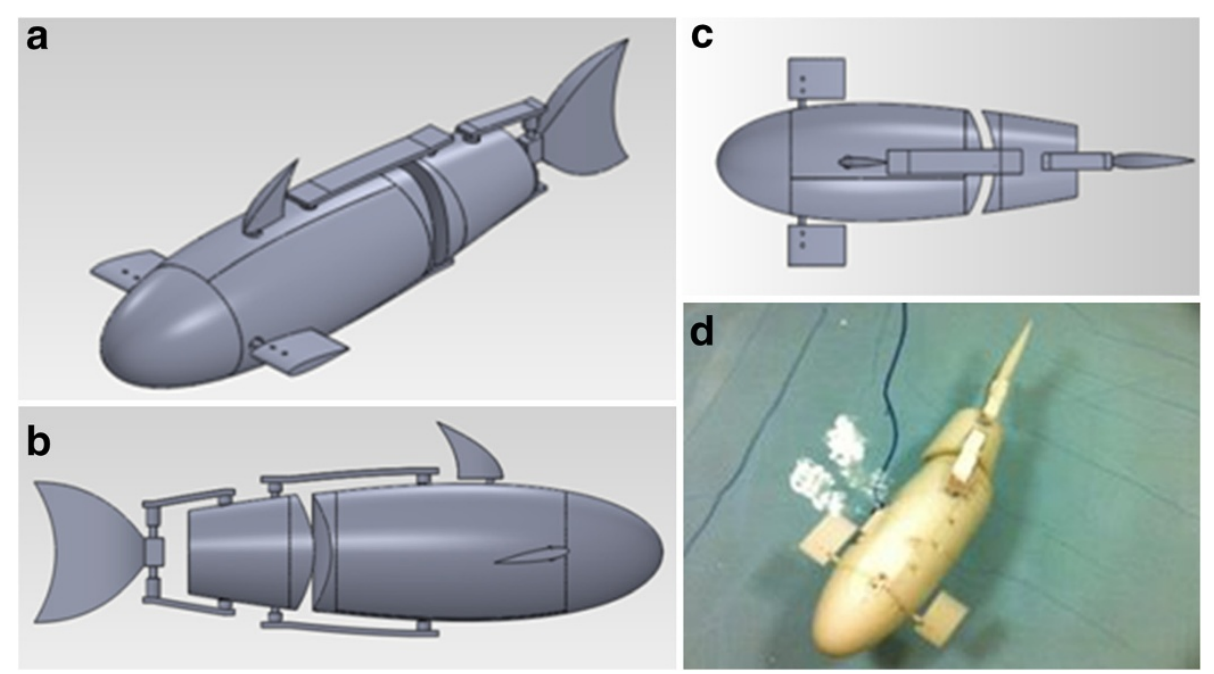

Figure 3 SolidWorks model and hardware prototype of the robotic fish. (a-c) Kinematics model of robotic fish with coordinated motion of multiple propulsive mechanisms. (d) Real hardware prototype used for closed-loop experiments.

forms that can be represented, the equation structure resembles primarily that of a time-dependent harmonic oscillation wave. It is found that propulsion patterns generated by fishes are dependent on purpose like foodsearch, hunting, migrating, mating, etc. [14]. The body dimensions (size) evolved over the ages as well as a specific environment also plays major role in these patterns. Based on the detailed study of the biological attributes of fish undulatory propulsion as well as Lighthill postulation on the oscillatory motion, a novel approach is made to extend and evaluate different mathematical functions that can fit in the frame of Lighthill. The integration of Lighthill mathematical model with robotic fish inverse kinematics and dynamics model as shown in Figure 4. The platform to validate the present research is the robotic fish designed and fabricated in our laboratory. Also, it is significant from the point of view that an evolutionary (biological) trait can be studied through the bioinspired algorithms evolving from these functions, like different need based actions, for example, a minimum energy body motion to travel a fixed distance etc. Therefore, a variety of actions and environments in real world can be understood through this research on a robotic fish (machine world). We can term it as understanding organic evolution of fish swimming (a biological hypothesis) through bio-inspired machines (designed on principles of mathematics and physics) [15,16], in an inverse learning map. Another viewpoint presented through our 3 DOF robotic fish model is that, on varying this DOF, a proportionate variation can be seen in the resulting motion. For

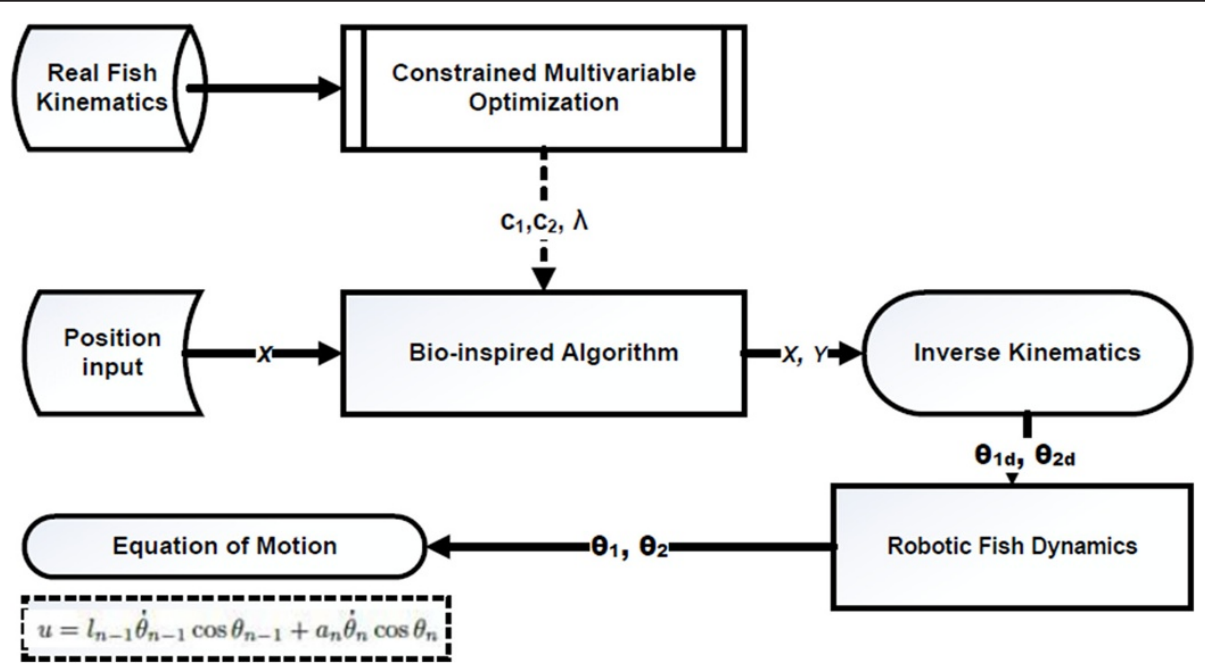

Figure 4 Block diagram showing integration of LH model in the robotic fish kinematics and dynamics model. 
example, selecting one DOF and a sine oscillating function would generate a tadpole motion in a 2D plane. Similarly, selecting one DOF and a suitable spline wave equation can produce a tadpole helical motion in $3 \mathrm{D}$ plane. On the other hand, if we introduce a 2 DOF and the Lighthill quadratic wave function, it results into a planar BCF carangiform (undulatory) motion. Further, adding a DOF and running the median pectoral fin (MPF) anguilliform waveform can lead to a more maneuvering motion resembling between a tuna and an eel. Therefore, combination of the various DOFs and Lighthill frame wave function can together attain as well as sustain different undulatory actions to undertake various tasks in different environments. The different mathematical wave functions are given below.

\section{Undulatory Lighthill quadratic amplitude body wave}

Lighthill was the one of the pioneers in applying the methods of slender body theory [13] to an undulating body swimming in an inviscid fluid medium. Dewar's [17] practical studies on kinematics and energetics on carangiform fishes by direct observations of biological fish are imminent to support Lighthill's theory as they lie in a digitized record. It was later used in one of the pioneering works of biologically inspired robot called MIT Robotuna [6] to validate a carangiform robotic swimming mode and other prominent research findings $[7,15]$. In this work, the mechanical robotic fish body generates a planar progressive wave function as a function of lateral curvature in spine and musculature, showing the undulatory propulsive behavior.

$$
y_{b o d y}(x, t)=\left(c_{1} x+c_{2} x^{2}\right)[\sin (k x+\omega t)]
$$

where $y_{\text {body }}$ is the transverse displacement of body, $x$ is the displacement along main axis, $k$ is the body wave number $(k=2 \pi / \lambda), \lambda$ is the body propulsive wave length, $c_{1}$ is the linear wave amplitude envelope, $c_{2}$ is the quadratic wave amplitude envelope, and $\omega$ is the body wave frequency. Taking this body wave, the present research does kinematic analysis to determine the proper body wave parameters $\left(c_{1}, c_{2}, k, \omega\right.$, etc.) for a desired and efficient undulatory swimming motion. We have further compared other mathematical functions in this frame (given below) to find their suitability to replace for a better undulatory wave function for robotic fish rectilinear swimming in a two-dimensional plane.

\section{Undulatory Lighthill cubic amplitude body wave}

In this paper, we propose the cubic body wave equation as an extension to Barrett's formulation by replacing the quadratic amplitude expression by a cubic spline polynomial. The motive behind this was to observe the efficacy of cubic polynomials in designing a continuous and smooth (non-jerky) motion through each rotational joint and therefore, passing more closely through the lateral displacement data points. It also accommodates more intermediate or via points in the plane. In the field of trajectory generation for robotics, cubic polynomials have been reported in majority due to its ability to track complicated trajectories efficiently [18].

$$
y_{b o d y}(x, t)=\left[\left(c_{1} x+c_{2} x^{2}+c_{3} x^{3}\right)\right][\sin (k x+2 \pi f \times t)]
$$

As the curvature of the wave primarily depends on the second derivative, it is found to be continuous here, to assist in velocity control. A major advantage of adding local intermediate points is not only to fit a smooth curvature for the harmonic motion but also flexibility to the body in physical terms. Throughout a given time period when the speed, orientation, and velocity changes gradually, these factors render the resulting trajectory smoothness. In physical terms, this smoothness means that there are lesser abrupt changes in power output for the robot's drive system, thus reducing navigational errors and helping to moderate the robot's energy consumption. Therefore, the function allows specifying undulatory motion that conserves both battery power while reducing travel time and minimizing navigational errors.

\section{Non-uniform rational B-spline (NURB) quadratic and cubic body wave (tadpole-like motion)}

In order to adapt the Lighthill frame harmonic waveform from control design perspective (with the presence of control points), a Bezier spline traveling waveform is proposed in this paper. Wu [19] has modified the methods of thin airfoil theory to analyze the motion of a waving $2 \mathrm{D}$ plate. The essential 2D and 3D methods developed by Lighthill and $\mathrm{Wu}$, respectively, have formed the basis from which the turning models were developed. Singh and Pedley [20] claim that Lighthill's classical elongated body theory for fish swimming forms the fundamental basis for the 3D flow model modified from the 2D small amplitude model reported earlier. Literature reports of combining efficient swimming propulsive force (speed) and maneuverability in the tadpole-like swimming mode can also be found [21]. The survey further mentions 3D propulsive motion of a free swimming larvae generating and controlling helical trajectory (rotation while swimming) by Long et al. [22]. Morphological and kinematic asymmetries of tail motion generate a rolling and pitching action [23] in addition to the yaw motion. Similar work, by Krishnamurthy et al. [24] find a bio-robotic implementation (robotic electric ray) in planar motion by modulating the propeller speed as the control variable, but the implementation was done in 2D (as a sub-case of 3D) to avoid dynamic complexity issues. Instantaneous trajectories are 
computed by extending Lighthill's two-dimensional model in xyz-plane by Singh and Pedley [20] and the tadpole's cycloptic helical motion [22]. Inspired by the aforesaid work, a framework was incubated and finally modeled for fitting the present robotic fish kinematic model, generating the 3D turning maneuvers. An attempt is made in this research also to define a mathematical expression in Lighthill frame for the 3D undulatory motion. It is to be noted that the robotic fish model is actuated by rotational joints with 1 DOF which can lead to various singularities in response to the mathematical solutions generated by the NURB trajectory. This motion would aim to satisfy the combined requirements of propulsive forces (speed) and turning angles (maneuverability) from the perspective of an energy efficient trajectory. This section, therefore, proposes and formulates a NURB mathematical equation as the new parametric curve to represent the propulsive wave behavior as lateral curvature in spine and musculature in $3 \mathrm{D}$. In addition to the control points, other advantages of using this model is that it can offer a common mathematical form for standard analytical shapes and also provide the flexibility to design a large variety of parametric shapes. Secondly, the geometrical evaluation can be reasonably fast by numerically stable and accurate algorithms. They are invariant under affine as well as perspective transformations. They are generalizations of nonrational B-splines and non-rational/rational Bezier surface curves. NURB shapes are not only defined by control points but also by their weights associated with each control point. This waveform assumes each reference point to be a control point as the traveling wave passes through only the first and the last control points therefore reducing the path length. This also adds to the trajectory planning in a way as the full control over the wave shape is achieved by tweaking a few parameters. They are also necessary to make it an optimal choice for the propulsive wave model. The weight calculation like parameter in other mathematical expressions is done based on the real kinematic data of carangiform fish. A NURB curve $C(u)$, which is also a vector-valued piecewise rational polynomial function representation, can be expressed as:

$$
C(u, v)=\frac{\sum_{i=1}^{n} \sum_{i=1}^{n}\left\{w_{i} \times P_{i} \times N_{i, k}(u, v)\right\}}{\sum_{i=1}^{n} \sum_{i=1}^{n}\left\{w_{i} \times N_{i, k}(u, v)\right\}}
$$

where $w_{i}$ is the weights, $P_{i}$ is the control points (vector), and $N_{i, k}$ is the normalized B-spline basis functions of degree $k$. The proposed mathematical model is in a normalized form. Therefore it reduces the scope of redundancy and dependency on one hand whereas increasing the consistency in results on the other. The proposed NURB mathematical model in Lighthill frame is verified for quadratic spline equations. To implement a curved trajectory, an arbitrary orientation for the robotic fish vehicle at its final position cannot be specified. To overcome this shortcoming, a somewhat more complicated curve in the form of a three-degree cubic polynomial is proposed, which uses an additional amplitude coefficient (weight) $c_{3}$ form. Like in the case of other two coefficients $c_{1}$ and $c_{2}$, its value is also dynamically calculated for the present robotic fish on the basis of the real fish study report [17]. Undulatory motions defined by the function in twodegree and three-degree NURB equations are written as:

$$
\begin{aligned}
& y_{b o d y}(x, t)=\left\{\frac{c_{1} x+c_{2} x^{2}}{\sqrt{c_{1}^{2}+c_{2}^{2}}}\right\} \sin (k x+2 \pi f \times t) \\
& y_{b o d y}(x, t)=\left\{\frac{c_{1} x+c_{2} x^{2}+c_{3} x^{3}}{\sqrt{c_{1}^{2}+c_{2}^{2}+c_{3}^{2}}}\right\} \sin (k x+2 \pi f \times t)
\end{aligned}
$$

The smooth transition it provides helps preserve momentum, reduce navigation error due to loss in the robot's drive mechanisms, and allow the robot to maintain its speed throughout its maneuvering. The proposed form (and its derivative) shown in the above equation has the advantage of being both continuous and differentiable at all points in the interval across all segments. The second derivative is also continuous throughout and differentiable across each interval except at 'via' points between segments. This form can permit considerable freedom in selecting the robot's orientation (turning) at via points. It is now computationally easier to calculate velocity, rotational velocity, and acceleration at via points throughout the trajectory, therefore, allowing us to specify maneuvers that can reduce travel time and minimizing navigational errors while conserving the battery power. The mathematical modification is implemented in the form of a transition from the existing uniform no-rational spline equation (Lighthill quadratic and cubic spline wave equations) to the NURB. As discussed earlier, another major implementation issue challenged by this improved model is to test the dynamic undulatory motion of the fish in a three-dimensional plane which may not be well-defined under the existing two-dimensional plane Lighthill linear/quadratic amplitude equations. Its efficacy in the control performance remains to be verified in the future work. But based on its properties [25] and results obtained in the present research, it is validated that the equation causes sudden rise around the initial conditions (jerk at start point) as shown in Figure 5b, if control points do not have a specific arrangement. Except for the two (quadratic and cubic) Bezier splines, other waveforms reveal smooth curvature indicating an undisturbed oscillatory/undulatory. This effect causes unwanted offset distance of desired control points from the actual trajectory points. 

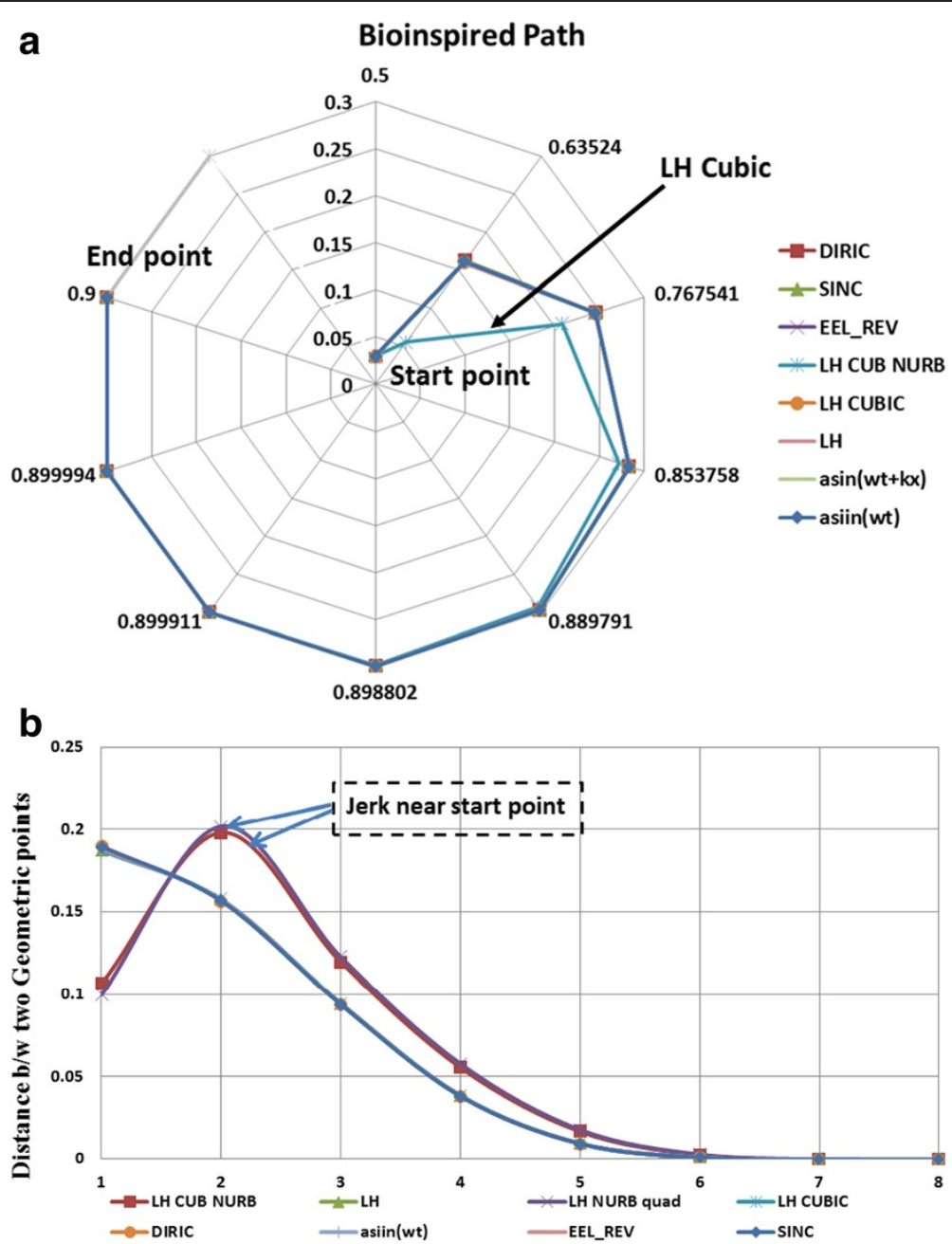

Figure 5 Time trajectory for different oscillatory/undulatory wave-functions. (a) Trajectory comparison graph for all waveforms. (b) Sudden rise around the initial conditions if control points do not have a specific arrangement.

\section{Undulatory SINC and DIRIC body waves}

The mathematical function called cardinal sine or sinc oscillates between positive and negative values with equal periodic repetition represents a sine wave that decays in amplitude as $1 / x$. This oscillation represents a decrease in amplitude with an increasing frequency. This also depicts that the mean value at a point $x \rightarrow 0$ will have a higher magnitude when compared to neighborhood of $\partial x \rightarrow 0+, \partial x \rightarrow 0$ - as well as for other oscillating peaks. The function is continuous at all real values with a removable singularity at $x=0$ (where first derivative is equal to 1 ). Figure 6 a shows two unnormalized (blue) and normalized (red) mathematical expressions. The normalization causes the definite integral of the function over the real numbers equal to 1 . It is a building block for a large function class in Fourier analysis (a major technique in the solution of differential equations). It is also seen to be a solution to the wave equations for transmission in communication theory. Physical meaning can be interpreted as a traveling wave signal propagated through body leading edge to the trailing edge for a sharp or gradual turning action. If we look into the traveling wave envelope proposed by Lighthill, sinc function suits into it such that it moves down the fish body with velocity $c$, and whose amplitude $c_{1} x+c_{2} x^{2}$ may vary with position along the fish body. The proposed undulating motion in Lighthill frame is given as:

$$
y_{b o d y}(x, t)=\left[\left(c_{1} x+c_{2} x^{2}\right)\right]\left\{\frac{\sin (k x+2 \pi f \times t)}{2 \pi f \times t}\right\}
$$

An extension to the sinc function, in the form of a Dirichlet function is defined in the Lighthill frame. This function is found to be a periodic sinc or aliased sinc function.

$$
y_{b o d y}(x, t)=f(x) \times h\left(t-\frac{x}{c}\right)
$$

Where 

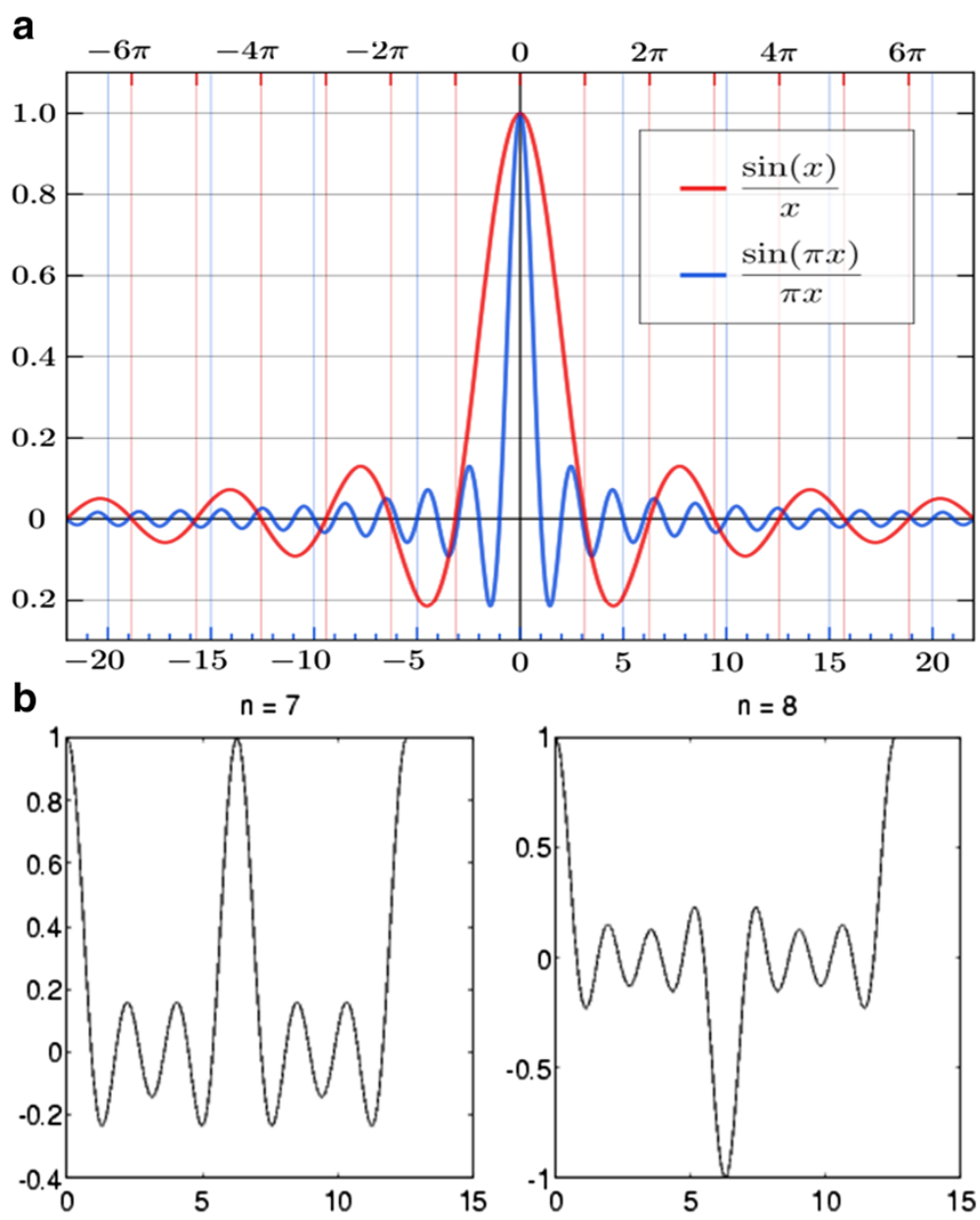

Figure 6 Undulatory cardinal sine traveling wave function. (a) Two unnormalized (blue) and normalized (red) mathematical expressions. (b) The tail beat of the caudal region as the traveling wave (midline) switches the amplitude mean value depending on the value of $N$.

$$
h(x, t)=\left\{\frac{\sin N(k x+2 \pi f \times t)}{N \sin (2 \pi f \times t)}\right\} .
$$

For $N$ as odd, the function has a period of 2; for $N$ as even, its period is 4 . It can be used to add orientation to the tail beat of the caudal region as the traveling wave (midline) switches the amplitude mean value depending on the value of $N$. It is verified in its discrete Fourier transform for an $\mathrm{N}$-point rectangular window as shown in Figure $6 \mathrm{~b}$. The centerline mathematical equation of motion in Lighthill frame is given by:

$$
y_{b o d y}(x, t)=\frac{1}{\left(c_{1} x+c_{2} x^{2}\right)}\left\{\frac{\sin (k x+2 \pi f \times t)}{\sin (2 \pi f \times t)}\right\}
$$

It can be noted that the quadratic wave amplitude is in the denominator and is used to dampen the oscillating wave function unlike the sinc function where it is allowed to grow as the numerator.

\section{Undulatory anguilliform body wave (EEL like/maneuvering model)}

Replicating the kinematics of a silver lamprey, a MPF anguilliform motion was proposed by Tytell [26]. From the maneuvering perspective, this equation adds another dimension to the overall fish propulsion. The motion of the fish centerline described by an exponentially growing traveling wave in Lighthill frame is given by a function as follows:

$$
y_{b o d y}(x, t)=\left[\left(c_{1} x+c_{2} x^{2}\right) \exp \left(2.18\left({ }^{x} / L-1\right)\right]\right.
$$

where $y$ is the lateral position of the midline, $x$ is the coordinate following the midline, $\alpha$ is the amplitude growth rate parameter [26], $L$ is the body length, and $\lambda$ is the propulsive wave length. Modifications were done in this equation as compared to the original equation due to the fact that the present robotic fish model is carangiform so the DOF is restricted to the body posterior (caudal) unlike anguilliform where the entire centerline participates in undulation. 
The idea of testing a modified anguilliform equation in a carangiform frame is whether maneuverability can be improved in slow undulatory motion. Two changes have been accommodated in order to undertake this test. Firstly, by replacing the constant amplitude by a quadratic amplitude wave and secondly, by provisioning that $\alpha$ can act as both an amplitude growth and dampening factor. This mathematical expression shows that a main wave function (sine or cosine) is decomposed in two Fourier functions. The primary function is again an oscillating sine wave which is multiplied by an exponentially growing function (can be represented in Fourier series) to satisfy the boundary conditions. The continuous everywhere exponential function is used for damping the sine wave. This function also resembles the Laplace transform with open unity integral.

\section{Results and discussion}

The implementation in robotic fish prototype for the Lighthill model verification and kinematic analysis is done in MATLAB@ $\odot$ and Simulink $\odot$ environment. Simulation results including time histories of forward velocity as well as the corresponding trajectories are discussed. Tests were done to show the effectiveness of Lighthill's propulsion model. Two conditions were compared, where the fish is allowed to travel a trajectory (using inverse kinematics algorithm) from an initial point to a destination point, without the Lighthill undulatory action as compared to the natural mode of its body motion, i.e., with Lighthill mathematical model implementation, fixed at three identified major parameters, i.e., oscillating (tailbeat) frequency $(\omega)$, propulsive wavelength $(\lambda)$ and caudal amplitude $\left(c_{1}\right.$ and $\left.c_{2}\right)$. For more clarity, Figure 7 shows the relative trajectory traversed with respect to center (origin) comparing the fish undulatory motion trajectory, with and without the Lighthill model implementation, clearly depicting the shorter route maintained by the Lighthill model over the non-Lighthill model. It therefore validates the classical theory postulated by Lighthill for the present robotic fish model. The above simulation shows the results and graphs; the performance factor of the robotic fish is calculated for a given (fixed) distance and battery power (onboard energy). It is found that with Lighthill implementation, the motion performance improves by $14.68 \%$. The motion performance (PI) is defined as follows:

$$
P I=\left[\frac{\text { distance }_{\text {w } / \text { LH }}-\text { distance }_{\text {with }} \text { LH }}{\text { total distance }}\right]
$$

The distance between two geometric points on a plane is calculated by the straight line equation algorithm:

$$
\text { distance }_{b / w p p}=\sqrt{\left(x_{i+1}-x_{i}\right)^{2}+\left(y_{i+1}-y_{i}\right)^{2}}
$$

The initial point is $x=0.5, y=0.0297$ and the final point is $x=0.9, y=0.3$. The coordinate points are normalized to a scale of 1: 100 (for distances measured in meters). The distance between these two points is found to be 0.482765046 units which is also the shortest distance (normal between the two planes). In the Lighthill quadratic wave equation, the amplitude constants $c_{1}$ and $c_{2}$ have been assumed to have a value [27] with little information on their choice. Our present research looks into the real kinematics of carangiform tuna fish in [17] as well as in the kinematic formulation of the Robotuna [17]. For two different conditions of caudal amplitude, the value of $\left(c_{1}, c_{2}\right)$ is calculated to be $0.002,0.00835$ and $0.2,0.01736$. For undulatory cubic spline, the values of $c_{3}$ are determined to be 0.01 and 0.346 under the two constraints. Tables 1 and 2 give the detailed information on the geometric points covered during the path traveled by the robotic fish. The paths are also plotted as trajectories as shown in Figure 8a,b,c,d,e,f,g,h. In Table 1, the trajectory details have been tabulated for wave function of oscillating sine, undulatory Lighthill quadratic, Lighthill cubic and NURB quadratic waves in the form of geometric points on the traveling plane of the robotic fish (undulating with these mathematical input wave functions). Their trajectories are plotted in Figure 8a,b,c,d. Comparing these trajectories with respect to the shortest distance, it is found that the percentage difference between the shortest path and user-defined wave generated path was least for undulatory Lighthill cubic polynomial with $1.2 \%$, followed by undulatory Lighthill quadratic polynomial with $1.22 \%$, and standing oscillating sine wave with $1.25 \%$; but a major deviation from the minimum value is observed in undulatory NURB quadratic wave with $4.19 \%$. In Table 2 , the trajectory details in the form of geometric points have been tabulated for mathematical input function of undulatory NURB cubic, SINC, DIRIC, and anguilliform waveforms. The trajectories drawn for these functions are shown in Figure 8e,f,g,h. It is noted that maximum percentage deviation from the shortest path continues with NURB cubic wave with $3.44 \%$ followed by SINC with $1.27 \%$, DIRIC with $1.28 \%$, and the lowest value (closest to the shortest distance) was with the anguilliform with $1.24 \%$. The user-defined wave functions have been analyzed, and some of them have been used to generate undulating traveling wave through the robotic fish body during closed-loop feedback operations. From Tables 1 and 2, it can be deduced that out of all the user-defined wave functions, the undulatory Lighthill cubic polynomial emerges out to be the most effective wave input to generate the trajectory with an improvement of $17.94 \%$ over a non-Lighthill path and an improvement of $1.1 \%$ over the Lighthill quadratic wave trajectory. It is closely followed by undulatory Lighthill quadratic polynomial 


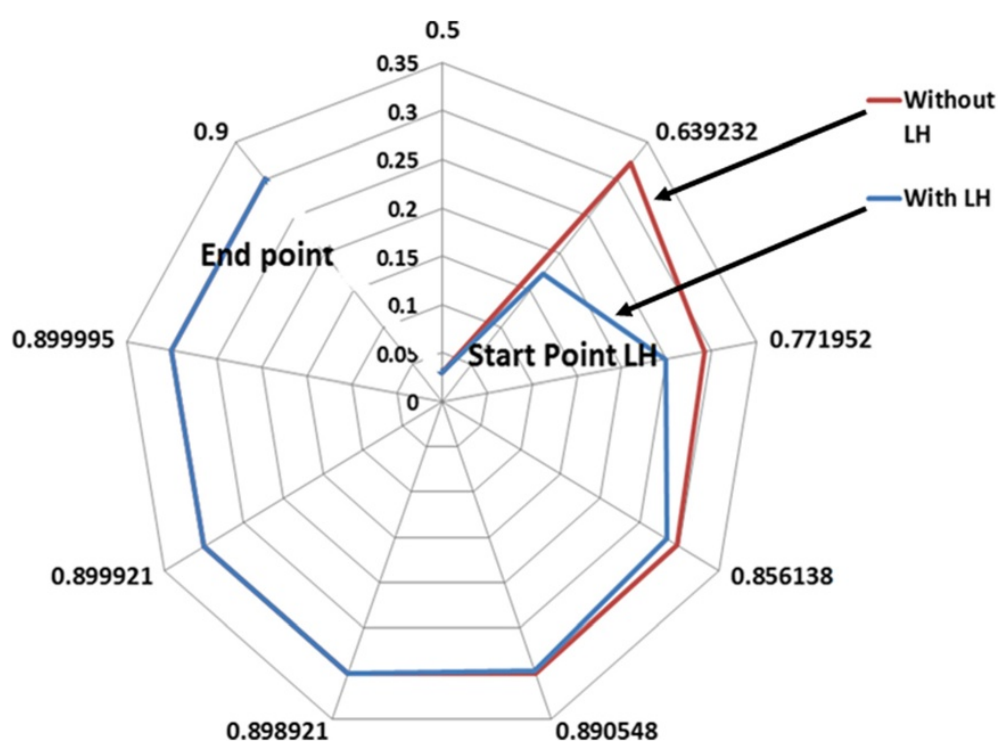

Figure 7 Relative path comparison relative to a center point (meters).

with $16.32 \%$ and anguilliform input with $14.7 \%$ mentioned in [26]. Plotting a trajectory comparison graph for all in Figure 5a, it is clear by looking into the blue line denoting the Lighthill cubic waveform that it generates as the path closest to the shortest distance between the two points of travel. During formulation, it has been strictly adhered that the function does not require computational complexity which will pose problem in real-time implementation. Another comparative study is undertaken relative to the primary Lighthill quadratic wave function. In Table 1, which mentions oscillating sine, Lighthill cubic, and NURB quadratic waveforms, (apart from Lighthill quadratic) it appears that only Lighthill cubic wave shows improvement, whereas, the two other wave functions namely oscillating fin and NURB quadratic wave stay close but record more than the Lighthill quadratic distance by $1.02 \%$ and $28.7 \%$, respectively. A similar comparison is undertaken for Table 2 .
It brings forth the fact that out of all the input functions mentioned in Table 2, NURB cubic wave shows maximum deviation from the Lighthill quadratic distance with $22.2 \%$ followed by DIRIC wave to be $6.4 \%$, SINC wave to be $5.8 \%$, and the closest to the ideal value is anguilliform wave with $2.2 \%$. Although all of them have shown a higher magnitude, w.r.t. Lighthill quadratic wave generated trajectory but some of them have managed to stay close to it. Therefore, two conclusions can be drawn from the complete analysis. Firstly, undulatory LH cubic waveform can prove to be an efficient algorithm for trajectory generation of a traveling propulsive body wave for robotic fish rectilinear motion. Secondly, other input functions proposed can also be used but mainly based on the required objective/action for, e.g., sinc or diric for turning or based on body dimensions for, e.g., NURB quad or cubic used for a tadpole-like motion. It can also be chosen on a purpose for, e.g., anguilliform/ eel-like for maneuvering and carangiform for speed. These

Table 1 Trajectory (geometric) points for mathematical oscillatory/undulatory propulsive waveforms

\begin{tabular}{|c|c|c|c|c|c|c|c|c|}
\hline \multirow[t]{2}{*}{ Node number } & \multicolumn{2}{|c|}{ Oscillatory sin } & \multicolumn{2}{|l|}{ LH quad } & \multicolumn{2}{|l|}{ LH cubic } & \multicolumn{2}{|c|}{ NURB quad } \\
\hline & $x$ pos & $y$ pos & $x$ pos & $y$ pos & $x$ pos & $y$ pos & $x$ pos & $y$ pos \\
\hline 1 (start point) & 0.500000 & 0.029700 & 0.500000 & 0.029700 & 0.500000 & 0.029700 & 0.500000 & 0.029700 \\
\hline 2 & 0.635240 & 0.160918 & 0.634913 & 0.159944 & 0.634781 & 0.159549 & 0.598402 & 0.046079 \\
\hline 3 & 0.767541 & 0.245599 & 0.767169 & 0.245283 & 0.767018 & 0.245155 & 0.723631 & 0.204411 \\
\hline 4 & 0.853758 & 0.283287 & 0.853553 & 0.283192 & 0.853471 & 0.283154 & 0.827144 & 0.270115 \\
\hline 5 & 0.889791 & 0.296484 & 0.889726 & 0.296460 & 0.889699 & 0.296450 & 0.880214 & 0.292851 \\
\hline 6 & 0.898802 & 0.299593 & 0.898791 & 0.299589 & 0.898787 & 0.299587 & 0.896969 & 0.298948 \\
\hline 7 & 0.899911 & 0.299970 & 0.899910 & 0.299969 & 0.899910 & 0.299969 & 0.899745 & 0.299912 \\
\hline 8 & 0.899994 & 0.299998 & 0.899994 & 0.299998 & 0.899994 & 0.299998 & 0.899982 & 0.299994 \\
\hline 9 (end point) & 0.900000 & 0.300000 & 0.900000 & 0.300000 & 0.900000 & 0.300000 & 0.900000 & 0.300000 \\
\hline
\end{tabular}


Table 2 Trajectory (geometric) points for mathematical oscillatory/undulatory propulsive waveforms

\begin{tabular}{|c|c|c|c|c|c|c|c|c|}
\hline & \multicolumn{2}{|c|}{ NURB cubic } & \multicolumn{2}{|l|}{ SINC } & \multicolumn{2}{|l|}{ DIRIC } & \multicolumn{2}{|c|}{ Anguilliform } \\
\hline & $x$ pos & $y$ pos & $x$ pos & $y$ pos & $x$ pos & $y$ pos & $x$ pos & $y$ pos \\
\hline 1 (start point) & 0.500000 & 0.029700 & 0.500000 & 0.029700 & 0.500000 & 0.029700 & 0.500000 & 0.029700 \\
\hline 2 & 0.603409 & 0.055233 & 0.635629 & 0.162066 & 0.635703 & 0.162282 & 0.635182 & 0.160746 \\
\hline 3 & 0.728721 & 0.208521 & 0.767983 & 0.245970 & 0.768066 & 0.246040 & 0.767476 & 0.245543 \\
\hline 4 & 0.830356 & 0.271654 & 0.853999 & 0.283398 & 0.854045 & 0.283419 & 0.853722 & 0.283270 \\
\hline 5 & 0.881458 & 0.293319 & 0.889869 & 0.296513 & 0.889884 & 0.296518 & 0.889780 & 0.296480 \\
\hline 6 & 0.897237 & 0.299041 & 0.898814 & 0.299597 & 0.898817 & 0.299598 & 0.898800 & 0.299592 \\
\hline 7 & 0.899771 & 0.299921 & 0.899912 & 0.299970 & 0.899912 & 0.299970 & 0.899911 & 0.299970 \\
\hline 8 & 0.899984 & 0.299995 & 0.899994 & 0.299998 & 0.899994 & 0.299998 & 0.899994 & 0.299998 \\
\hline 9 (end point) & 0.900000 & 0.300000 & 0.900000 & 0.300000 & 0.900000 & 0.300000 & 0.900000 & 0.300000 \\
\hline
\end{tabular}

mathematical inputs are responsible to build different bioinspired algorithms which later combine with the realtime kinematics and dynamics of the robotic fish. Henceforth, the robotic fish driven by these algorithms support adaptation policy shown by a biological fish in different environments.

\section{Operating region}

Using the system dynamic model formulated as Equation (3), MATLAB simulation result are generated for the robot closed-loop motion between two points in a three-dimensional plane. This mechanism uses a bioinspired algorithm under Lighthill cubic wave function as it is found to be the best result for trajectory generation. The simulation results $[28,29]$ are useful during the closed-loop experimental verification [9] and operation of the robotic fish prototype as we know the variation of forward velocity with major kinematic parameters like tailbeat frequency (TBF), caudal amplitude (CA), propulsive wavelength (PW), and yaw angle. To enhance the system repeatability therefore reliability, each parameter is plotted for various wavelengths and operating tail-beat frequency values, resulting in the operating region. Based on the simulation results discussed above, an ORE [30] that summarizes these major parameters in consideration is formed in the form of a plot as shown in Figure 9a,b. The vertical axis in usual notation mentions swimming (forward) velocity in $\mathrm{cm} / \mathrm{s}$ while the horizontal axis is denoted by two parameters, TBF and PW. The range of TBF is from 0 to 2.8 units $(\mathrm{Hz})$ while for $P W$ it is from 1.2 to 2.2 units $(\mathrm{m})$. Variation of swimming speed with TBF and PW is shown as oscillating bell-shaped curves with circle and square markers, respectively. The two peaks marked by a star (with close dashed lines) symbol and a triangle (with wide dashed lines) symbol show the peak velocities achieved for a given value of TBF $(1.1 \mathrm{~Hz})$ and PW $(1.45 \mathrm{~cm})$, respectively. It is to be observed that for the peak velocity of $17.35 \mathrm{~cm} / \mathrm{s}$, the choice of TBF is unique, i.e., $1.1 \mathrm{~Hz}$; but there is no wavelength available in the permissible range.
Contrary to this, if the peak velocity of $17.3 \mathrm{~cm} / \mathrm{s}$ (triangle symbol) is chosen, for a unique value of propulsive wavelength at $1.45 \mathrm{~cm}$, there exist two TBF values at 1 and $1.15 \mathrm{~Hz}$. Therefore, for any choice of speed (based on the purpose like fast or moderate swimming), the combinational choice of the two parameters can be found for robotic fish. Interestingly, it can be observed that most of the times, for a given velocity, there exists two or more values of TBFs or PWs. This is due to the non-linear nature of the curve or many one function mapping. The final choice by the biological fish must be done based on the metabolic rates (related to Froude efficiency) or any other hidden parameter, which is our future course of study. The value of caudal amplitude is kept constant at $c_{1}=$ 0.002 and $c_{2}=0.00835$. Another plot, Figure 9c from Dewar's experiment on the biological equivalent (yellowfin tuna), shows the results of swimming velocity $(\mathrm{cm} / \mathrm{s})$ versus time. This plot also shows metabolic rate (triangular marker) trend with time as the fish moves. The swimming velocity pattern and magnitude are in agreement with the present robotic fish kinematic reports. This suggests that the kinematic design of present robotic fish has somewhat successfully emulated a real fish. Clearly, the kinematic studies relation of the four parameters (TBF, CA, PW, and yaw angle) for the present robotic fish underwater vehicle model is validated at par with Dewar's findings [17]. Looking from another dimension $[16,24]$, this can be seen as an important and useful research to understand the biology of fish swimming, with applied mechanics replacing fish dynamics and arithmetic formulation substituting for the undulatory input motion, respectively. Robotic fish can therefore be used to study the fish biology since many parametric results were found to be in agreement with test data of tropical yellowfin tuna. The present ORE patterns therefore can also allow us to make predictions closer to the real fish kinematics. Another objective of using the ORE is to implement the closed-loop [9] control of the robotic fish with the help of comprehensive kinematic study undertaken the w.r.t. parameters. This would also make it 

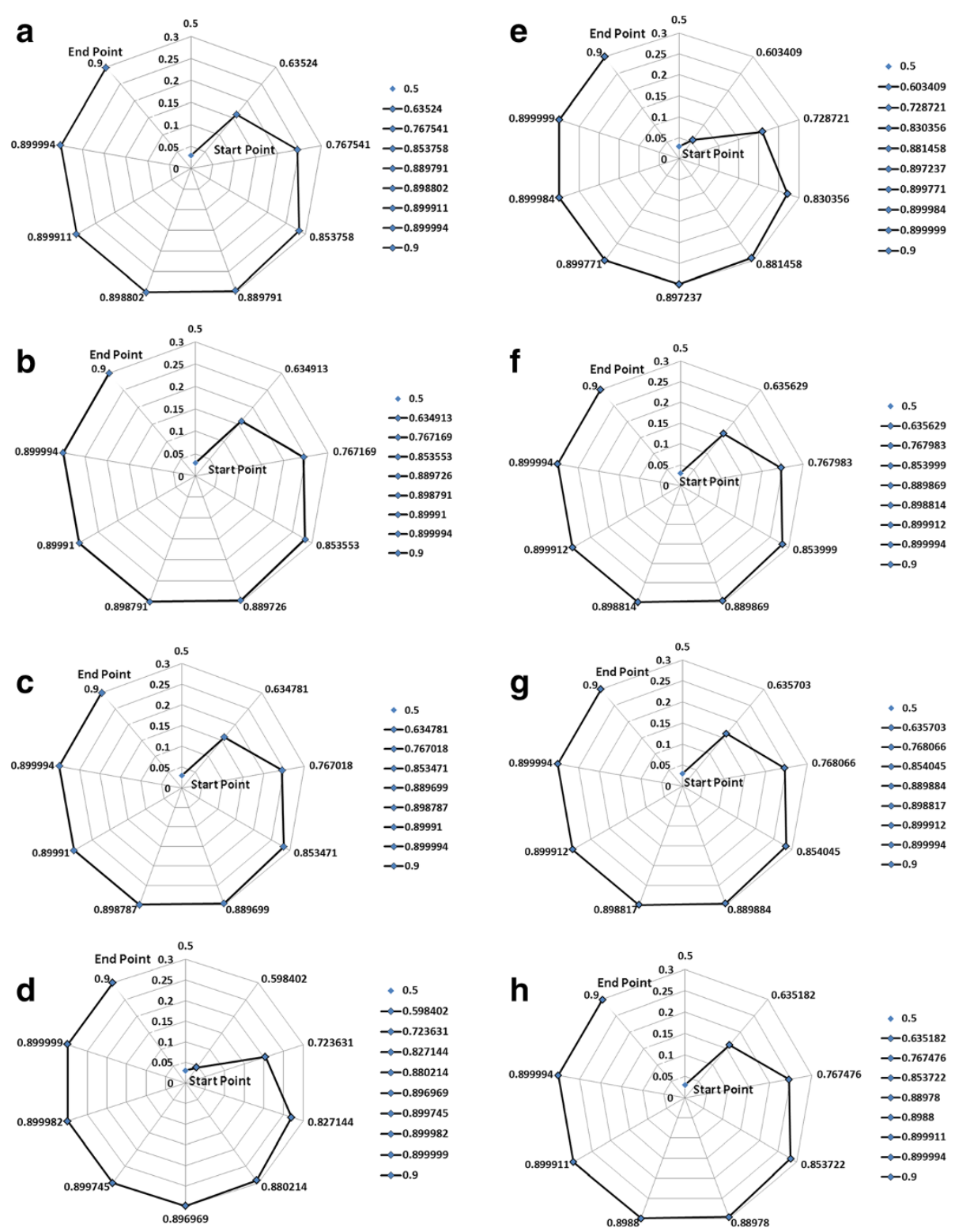

Figure 8 Trajectory traversal between two fixed points using different oscillatory/undulatory wave functions. (a-d) Plotted trajectories for wave function of oscillating sine, undulatory Lighthill quadratic, Lighthill cubic, and NURB quadratic wave in the form of geometric points on the traveling plane of the robotic fish. (e-h) Trajectory details in the form of geometric points tabulated for mathematical input function of undulatory NURB cubic, SINC, DIRIC, and anguilliform waveforms.

easier to operate the robot and less time consuming as the kinematic characteristic of robotic fish is presented in the form of a simple operating region chart [30]. This study stresses on the importance of major kinematic parameters while neglecting the minor parameters to avoid computational complexity. Present kinematic study does a value addition to the closed-loop control $[9,31]$ technique as it discusses intriguing yet solvable arithmetic formulations (of input waveforms) in Lighthill frame to run a sophisticated biological process (fishlike undulatory/oscillatory motion). The simulation results also denote that amplitude and wavelength are found to be the less chosen parameters compared to the TBF if swimming speed is considered. Also a compromise (optimization) of these three important parameters is required for the most efficient undulatory fish body motion. Clearly, the relation of distance-based performance factor and forward velocity for the present robotic fish underwater vehicle model is validated at par with Dewar's findings [17]. The yaw axis servomotor is a HiTec HS-5646WP (11.3 Kg-cm/6 V) used in all the joints. Arduino-Uno with ATMEGA2560 serves as the central processing unit for the robotic fish. The mechanical 

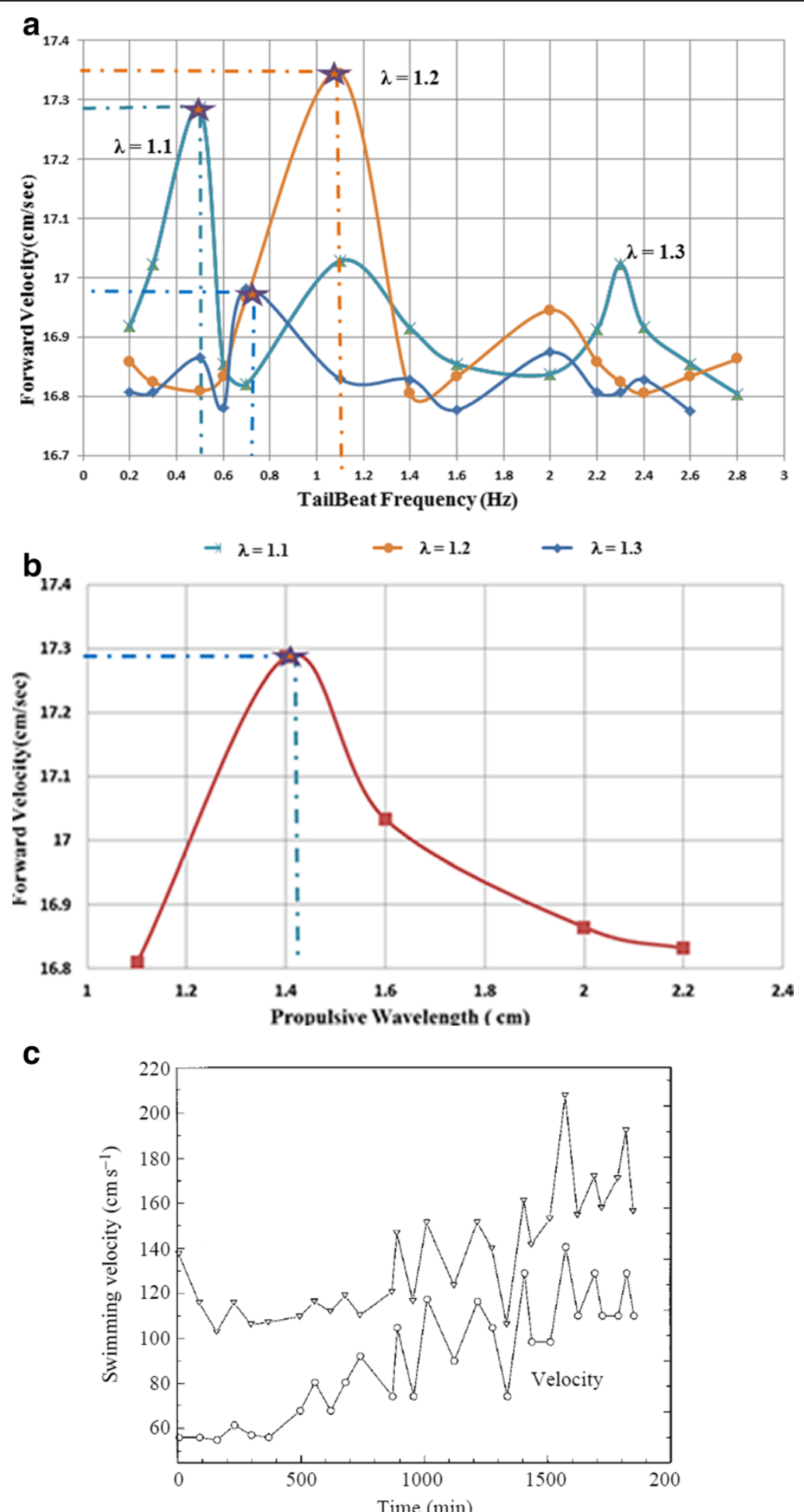

Figure 9 Operating region for tail beat frequency (TBF) and propulsive wavelength.

computer-aided (CAD) design and animation has been implemented with the help of the Solidworks (mechanical model) and MATLAB SimMechanics VRML (3D motion). The motivation of this paper is described by the remarkable swimming abilities of BCF mode carangiform style of swimming built as a robotic fish prototype. The NACA airfoil aerodynamic structure has been designed to boost the swimming efficiency by reducing the 
drag. Using the present dynamic model and derived steady kinematic simulation results $[28,29]$, the closed-loop experiments [9] were done for different body motion configurations to emulate the undulation of the robotic fish in fluid environment shown in Figure 10.

\section{Conclusions}

The present research has focused on the relevance of Lighthill (LH) based biomimetic robotic propulsion of a proposed 2-joint, 3-link multibody vehicle model, biologically inspired by a BCF carangiform fish. The objective of this paper is to translate the BCF mode carangiform swimming behavior of a biological fish to a robotic fish to allow its energy efficient navigation over a given distance using a good balance speed and agility characteristics. The robotic fish model (kinematics and dynamics) is integrated with the LH mathematical model framework. Mathematical input waveforms are investigated in LH framework to generate posterior body undulatory movements. These functions are combined with robot inverse kinematics to generate various bio-inspired trajectories for the posterior robotic fish vehicle motion. Distance-based performance criteria for a given trajectory are proposed to do a comparative analysis for the input undulatory waveforms. Comparisons are done between a non-LH and a path defined in LH frame. Based on present kinematic model simulation of identified kinematic parameters, closed-loop experiments are done to establish operating region for two critical kinematic parameters TBF and PW. This finding also aims to facilitate future experiments for a robotic fish model. Interestingly, the robotic behavior in simulation and experiments (closed-loop) is showing swimming behavior similar to the biological fish mentioned by Lighthill slender body theory and Dewar's kinematic experiments on yellow fin tuna. The future work primarily focuses on the development of an improved inverse kinematics algorithm within the Lighthill framework. A behavior-based control strategy and its implementation for energy efficient undulatory fish motion would further strengthen the vision of the machines mimicking biology principles in a significant way.

\section{Appendix A}

(a) Link and joint parameters (shown in Figure 2)

- Joint angle $\left(\theta_{i}\right)$ : the angle of rotation from the $X_{i-1}$ axis to the $X_{i}$ axis about the $Z_{i-1}$ axis. It is the join variable if joint $i$ is rotary.

- Joint distance $\left(d_{i}\right)$ : the distance from the origin of the (i-1) coordinate system to the intersection of the $Z_{i-1}$ axis and the $X_{i}$ axis along the $Z_{i-1}$ axis.

- Link length $\left(a_{i}\right)$ : the distance from the intersection of the $Z_{i-1}$ axis and the $X_{i}$ axis to the origin of the $i_{t h}$ coordinate system along the $X_{i}$ axis.

- Link twist angle $\left(\alpha_{i}\right)$ : the angle of rotation from the $Z_{i-1}$ axis to the $Z_{i}$ axis about the $X_{i}$ axis.

(b)Reference Frames

- $F_{I}$ : inertial frame of manipulator-based system.

- $F_{B}$ : base frame located at the center of mass of the base.

- $F_{i}$ : coordinate frame of the $i_{t h}$ link of the system.

\section{(c) Vectors}

- $r_{B}$ : position of frame $F_{O}$ relative to and projected onto frame $F_{B}$.

- $r_{I}$ : position of frame $F_{O}$ relative to and projected onto frame $F_{O}$.

- $d_{i}$ : position of frame $F_{i}$ relative to and projected onto frame $F_{O}$.

- $r_{i}$ : position of point on link $i$ relative to frame $F_{O}$.

- $i$ : position of point on link $i$ relative frame $F_{i}$.

- $b$ : position of point on the base relative frame $F_{B}$.
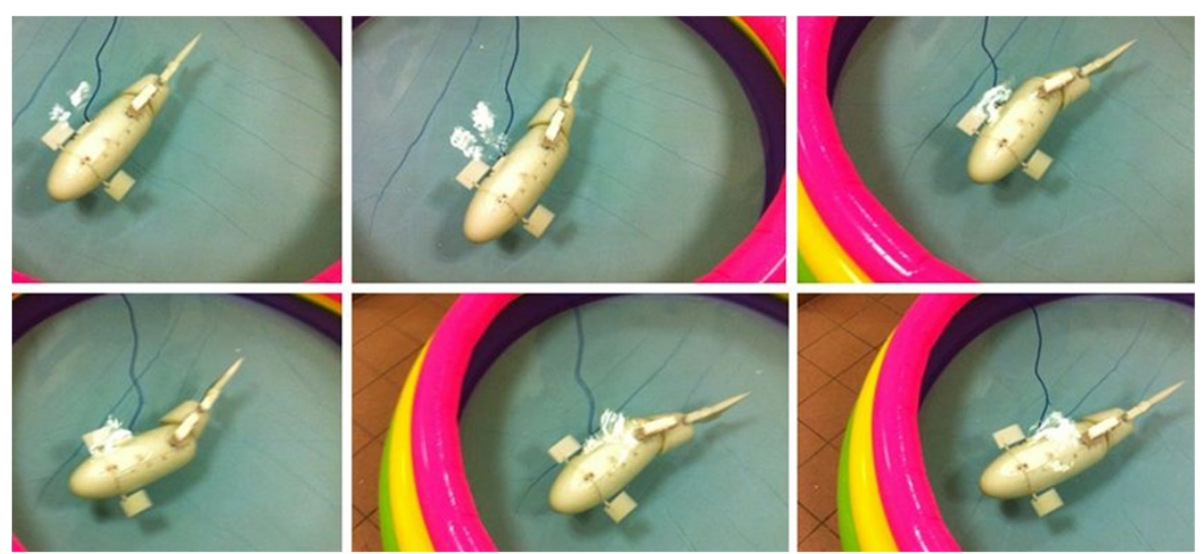

Figure 10 Closed-loop hardware prototype motion in different frames. 
- $b_{B}$ : position of point on the base relative to frame $F_{I}$.

- $v_{i}$ : velocity of point on link $i$ relative to frame $F_{I}$.

- $v_{B}$ : velocity of point on the base relative to frame $F_{I}$.

\section{Competing interests}

The authors declare that they have no competing interests.

\section{Authors' contributions}

ARC has conceptualized and written the manuscript with contributions from VK, BP, and RK SKP has rendered critical comments and revised the manuscript. All authors have contributed to the final manuscript.

\section{Acknowledgements}

We would like to thank Mr. Alok Agrawal of Purdue University for his key contributions for the mechanical CAD design of the robotic fish. We would also like to acknowledge the useful suggestions and feedback given by Mr. Shailabh Suman of Acoustic Research Lab NUS, Dr. Mandar Chitre of Acoustic Research Lab NUS, Dr. Pablo Alvaro Valdivia of Singapore-MIT Alliance for Research and Technology (SMART), Professor Marcelo H. Ang Jr. and Professor Xu Jianxin of Department of Electrical and Computer Engineering We thank the Office of Defense Science and Technology Agency (DSTA) for their support of the present research.

\section{Funding}

This work is presently supported by the Defense Science and Technology Agency (DSTA), under the Ministry of Defense (Singapore), Government of Singapore under Grant R-263-000-621-232-MINDEF-DSTA for Underwater Vehicle Technology Project STARFISH.

\section{Author details}

${ }^{1}$ Department of Electrical and Computer Engineering, National University of Singapore, Singapore 117576, Singapore. Electrical Engineering Department, Malviya National Institute of Technology, Jaipur, India.

Received: 9 July 2014 Accepted: 7 October 2014

Published online: 20 November 2014

\section{References}

1. Triantafyllou MS, Techet AH, Hover FS (2004) Review of experimental work in biomimetic foils. IEEE J Ocean Eng 29:585-594. doi:10.1109/JOE.2004.833216

2. Bullinaria JA (2003) From biological models to the evolution of robot control systems. Phil Trans R Soc A 361:2145-2164. doi:10.1098/rsta.2003.1249

3. Bandyopadhyay PR (2005) Trends in biorobotic autonomous undersea vehicles. IEEE J Ocean Eng 30:109-139. doi:10.1109/JOE.2005.843748

4. Lighthill MJ (1970) Aquatic animal propulsion of high hydro-mechanical efficiency. J Fluid Mech 44:256-301. http://dx.doi.org/10.1017/ S0022112070001830

5. Sfakiotakis M, Lane DM, Davies JBC (1999) Review of fish swimming modes for aquatic locomotion. IEEE J Ocean Eng 24:237-252. doi:10.1109/48.757275

6. Barrett D, Grosenbaugh M, Triantafyllou MS (1996) Optimal control of a flexible hull robotic undersea vehicle propelled by an oscillating foil. IEEE Symposium on Autonomous Underwater Technology, Monterey, CA, pp 1-9, doi:10.1109/AUV.1996.532833

7. Junzhi Y, Min T, Shuo W, Erkui C (2004) Development of a biomimetic robotic fish and its control algorithm. IEEE Trans Syst Man Cybern B Cybern 34:No. 4

8. Motomu N, Norifumi O, Kyosuke O (2003) A study on the propulsive mechanism of a double jointed fish robot utilizing self-excitation control. JSME Int J Series C 46:982-990. doi:10.1299/jsmec.46.982

9. Roy Chowdhury A, Prasad B, Kumar V, Kumar R, Panda SK (2014) Inverse dynamics kinematic control of a bio-inspired robotic-fish underwater vehicle propulsion based on Lighthill's slender body model. IEEE OES MTS OCEANS, Taipei, Taiwan

10. Fossen $\mathrm{Tl}$ (2011) Handbook of marine craft hydrodynamics and motion control. John Wiley \& Sons. doi:10.1002/9781119994138

11. Fu KS, Gonzalez RC, Lee CS (1987) Robotics control, sensing, vision and intelligence. McGraw-Hill New York
12. Roy Chowdhury A, Prasad B, Kumar V, Kumar R, Panda SK (2014) Bioharmonized dynamics model for a biology inspired carangiform robotic fish underwater vehicle. In: Proc. 19th IFAC World Congress, Vol. 19. Cape town

13. Lighthill MJ (1960) Note on the swimming of slender fish. J Fluid Mech 9:305-317. http://dx.doi.org/10.1017/S0022112060001110

14. Videler JJ (1993) Fish Swimming. Chapman and Hall, London. doi:10.1007/ 978-94-011-1580-3

15. Liu J, Huosheng $H$ (2010) Biological inspiration: from carangiform fish to multi-joint robotic fish. J Bionic Eng 7(1):35-48

16. Long JH Jr (2011) Biomimetics_robotics based on fish swimming. Chapter 244. In: Encyclopedia of fish physiology: from genome to environment. Elsevier Academic Press, San Diego, CA, ebook isbn: 9780080923239

17. Dewar H, Graham J (1994) Studies of tropical tuna swimming performance in a large water tunnel-kinematics. J Exp Biol 192:45-59

18. Thompson S, Koto Kagami S (2005) Continuous curvature trajectory generation with obstacle avoidance for car-like robots. IEEE International Conference on Computational Intelligence for Modeling, Control and Automation:863-870, doi:10.1109/CIMCA.2005.1631373

19. Wu T (1961) Swimming of a waving plate. J Fluid Mech 10:321-344, http://dx.doi.org/10.1017/S0022112061000949

20. Singh K, Pedley TJ (2008) The hydrodynamics of flexible-body maneuvers in swimming fish. Physica D: Nonlinear Phenom 237(1417):2234-2239, http://dx.doi.org/10.1016/j.physd.2008.02.002

21. Liu H, Kawachi K (1999) A numerical study of undulatory swimming. J Comput Phys 155:223-247, doi:http://dx.doi.org/10.1006/jcph.1999.6341

22. Long JH, Jr, Lammert AC, Pell CA, Kemp M, Strother J, Crenshaw HC, McHenry MJ (2004) A navigational primitive: biorobotic implementation of cycloptic helical klinotaxis in planar motion. IEEE J Ocean Eng 29:795-806. doi:10.1109/JOE.2004.833233

23. Chen B, Jiang SR, Liu YD (2010) Research on the kinematic properties of a sperm like swimming micro robot. J Bionic Eng 7:123-129, http://dx.doi.org/ 10.1016/S1672-6529(09)60225-0

24. Krishnamurthy $P$, Khorrami F, de Leeuw J, Porter M, Livingston K, Long JH Jr (2010) An electric ray inspired biomimetic autonomous underwater vehicle. American Control Conference 2010:5224-5229, INSPEC Accession Number: 11509303

25. Bock RK, Krischer W (1998) Data analysis briefbook. Springer, Berlin. doi:10.1007/978-3-662-03725-6

26. Tytell ED (2004) The hydrodynamics and eel swimming II: effects of swimming speed. J Exp Biol 207:3265-3279. doi:10.1242/jeb.01139

27. Zhou C, Low KH, Chong CW (2009) An analytical approach for better swimming efficiency of slender fish robots based on Lighthill's model. IEEE International Conference on Robotics and Biomimetics ROBIO:1651-1656, doi:10.1109/ROBIO.2009.5420418)

28. Roy Chowdhury A, Prasad B, Kumar V, Kumar R, Panda SK (2012) Implementation of a BCF mode bio-mimetic robotic fish underwater vehicle based on Lighthill mathematical model. IEEE ICROS International Conference on Control, Automation and Systems Jeju, Korea:437-442, (INSPEC Accession Number: 13225528). (Best Student Paper Award)

29. Roy Chowdhury A, Prasad B, Kumar V, Kumar R, Panda SK (2012) Kinematics study and implementation of a biomimetic robotic-fish underwater vehicle based on Lighthill slender body model. IEEE OES Autonomous Underwater Vehicles (AUV), National Oceanography Centre, Southampton UK:1-6, doi:10.1109/AUV.2012.6380721

30. Roy Chowdhury A, Prasad B, Kumar V, Kumar R, Panda SK (2013) Finding an operating region for a bio-inspired robotic fish underwater vehicle in the Lighthill framework. IEEE International Conference on Robotics and Biomimetics ROBIO Shenzhen China, 854 - 860, doi:10.1109/ROBIO.2013.6739569

31. Roy Chowdhury A, Panda SK (2014) Kinematic parameter based behavior modelling and control of a bio-inspired robotic fish. Proceedings of SICE Annual Conference 2014, Sapporo, Japan

\section{doi:10.1186/s40638-014-0015-2}

Cite this article as: Chowdhury et al:: Kinematic study and implementation of a bio-inspired robotic fish underwater vehicle in a Lighthill mathematical framework. Robotics and Biomimetics 2014 1:15. 